\title{
Active stabilization of unmanned aerial vehicle imaging platform
}

(C) The Author(s) 2020

Article reuse guidelines: sagepub.com/journals-permissions DOI: $10.1177 / 1077546320905494$ journals.sagepub.com/home/jvc

(S)AGE

\author{
Mohit Verma ${ }^{1,2} \odot$, Vicente Lafarga', Mael Baron' and \\ Christophe Collette ${ }^{1,3}$
}

\begin{abstract}
The advancement in technology has seen a rapid increase in the use of unmanned aerial vehicles for various applications. These unmanned aerial vehicles are often equipped with the imaging platform like a camera. During the unmanned aerial vehicle flight, the camera is subjected to vibrations which hamper the quality of the captured images/videos. The highfrequency vibrations from the unmanned aerial vehicle are transmitted to the camera. Conventionally, passive rubber mounts are used to isolate the camera from the drone vibrations. The passive mounts are able to provide reduction in response near the resonance. However, this comes at the cost of amplification of response at the higher frequency. This article proposes an active vibration isolation system which exhibits improved performance at the higher frequencies than the conventional system. The active isolation system consists of a contact-less voice coil actuator supported by four springs. Experiments are carried out to study the effect of vibrations on the quality of images captured. The characterization of drone vibrations is also carried out by recording the acceleration during different flight modes. The performance of the proposed isolation system is experimentally validated on a real drone camera subjected to the recorded drone acceleration spectrum. The isolation system is found to perform better than the conventional rubber mounts and is able to reduce the vibrations to a factor of one-fourth. It can be effectively used to improve the image acquisition quality of the unmanned aerial vehicles.
\end{abstract}

\section{Keywords}

Active stabilization, vibration isolation, drone, voice coil actuators, camera

\section{Introduction}

Over the last few decades, the use of unmanned aerial vehicles (UAVs) has expanded to various domainsautonomous vehicles (Jin et al., 2000), military surveillance (Ma'sum et al., 2013), precision agriculture (Chen et al., 2002), remote sensing (Xiang and Tian, 2011), aerial photography (Boike and Yoshikawa, 2003), optical payloads (Yao and Jingrui, 2015), and vision-based health monitoring of civil structures (Wahbeh et al., 2003). UAVs are most often equipped with the imaging platform, which capture images during its flight. The images collected are then compiled and analyzed to arrive at the data useful for monitoring and decision-making. Unlike satellite, the images collected by UAVs are far more localized and have higher resolution. Moreover, image acquisition using UAVs is far more economical than satellite imagery. Multi- and hyper-spectral cameras can also be combined with UAVs to capture images beyond visible light range. The extraction of useful information largely depends on the quality of the images captured by the UAV. During flight, the imaging platform is subjected to disturbances of varying amplitudes and frequencies. These disturbances if not controlled or corrected can result in blurred images. Various methods of image stabilization have been developed over the years. The methods for image stabilization are broadly classified into three main categories - optical, digital, and mechanical image stabilization (Baek, 2012; Levoy, 2014). Different types of vibrations encountered by UAVs during its course of flight are shown in Figure 1(a). Figure 1(b) shows the methods of image stabilization suggested by Windau and

\footnotetext{
'BEAMS Department, Precision Mechatronics Laboratory, Université Libre de Bruxelles, Belgium

${ }^{2} \mathrm{CSIR}$ - Structural Engineering Research Centre, India

${ }^{3}$ Department of Aerospace and Mechanical Engineering, University of Liège, Belgium

Received: I October 2019; accepted: 9 January 2020
}

\section{Corresponding author:}

Mohit Verma, ASTaR Laboratory, CSIR - Structural Engineering Research Centre, CSIR Madras Campus, Taramani, Chennai 600II3, Tamil Nadu, India.

Email: mohitverma@serc.res.in 
Itti (2011) in the frequency-amplitude domain. It can be observed from Figure 1(b) that only mechanical image stabilization has the ability to perform well in the entire domain. Gimbals are used to compensate large-amplitude low-frequency vibrations. Vibration isolation mounts are used for compensating low-amplitude high-frequency vibrations.

The mechanical image stabilization based on passive systems is purely mechanical. Passive rubber mounts are conventionally used to reduce the effect of high-frequency vibrations on the camera. Kienholz et al. (1996) used a magnetically damped vibration isolator for large optical payloads. The isolator was in the form of a strut having a titanium coil spring and a passive damper, which dissipates energy through the eddy current. Gjika and Dufour (1999) developed a dry friction isolator for infrared cameras mounted on a helicopter. Inspired by the avian's

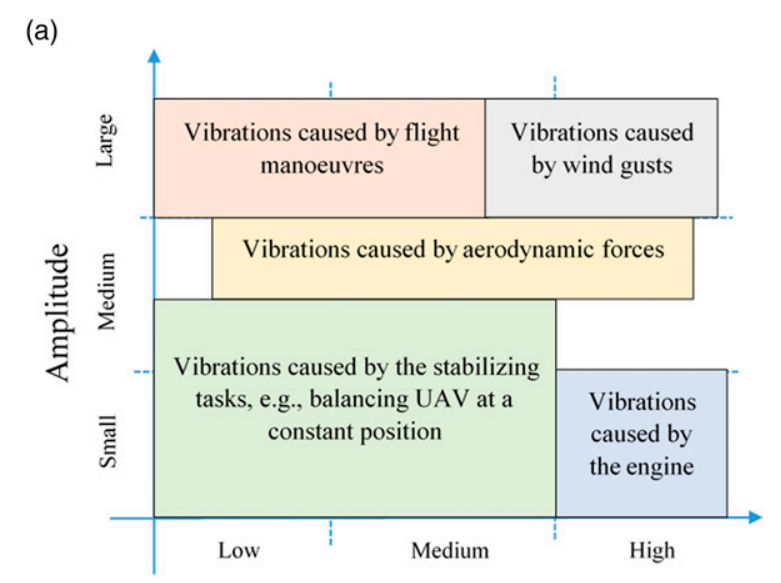

Frequency

(b)

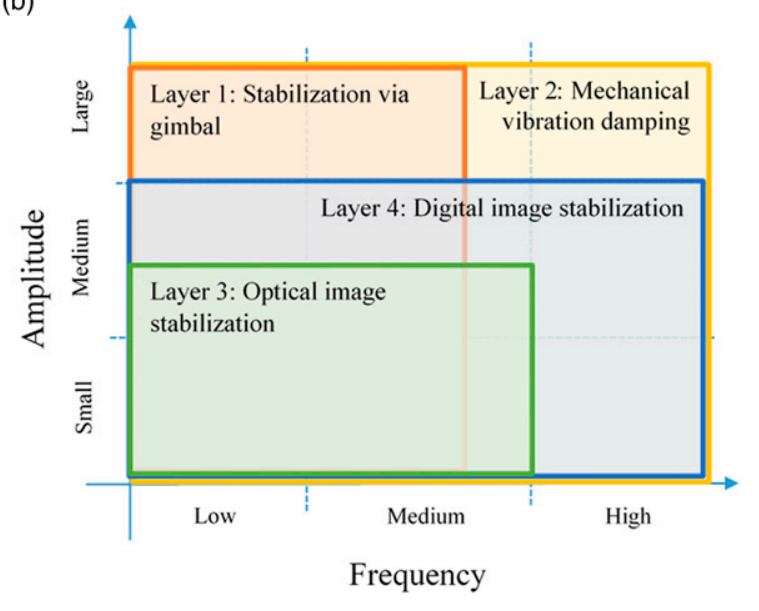

Figure I. Frequency-amplitude coverage: (a) vibrations in unmanned aerial vehicle and (b) suggested stabilization method. Notice that mechanical stabilization is effective for wide range of frequency. Gimbal is used for compensating low-frequency vibrations, whereas high-frequency vibrations are compensated using passive/active isolation. ability to keep its head steady with respect to the horizon, a passive system was proposed by approximating the neck of the bird with linear mass-spring-damper system (Pete et al., 2015). The system was found to attenuate the large body oscillations. Such type of passive system is ideal for the bird-inspired drones with the flapping mechanism. Webster and Semke (2005) developed an isolation system for camera for remote sensing application. The system proposed was a combination of vibration isolator and absorber. Viscoelastic materials were incorporated to provide damping and isolation to the system. The frequency-dependent behaviour of the viscoelastic material was found to give better results than the conventional system using springs and dampers. Maes et al. (2014) presented wire rope isolators for the camera system mounted on the body of the aircraft.

Although passive mounts are effective in reducing the vibration near resonance, it comes at the cost of amplification at high frequency. Active isolation can overcome the drawbacks of the passive system. The concept of active isolation for UAVs is shown in Figure 2. Many researchers developed mounts using piezoelectric actuators (Stuckel and Semke, 2011; Stuckel et al., 2011). Oh et al. (2010, 2011) and Park and Choi (2008) presented a camera mounting system using piezo stack actuators. The mount consisted of a rubber element, an inertial mass, and a piezoelectric actuator. The inertial mass was driven by the actuator. A sliding mode controller was used, which can handle the model uncertainties and the hysteretic behaviour of the actuator. Hardware-in-loop simulation was used to evaluate the performance of the mount. Baer and Semke (2012) developed a frictionless vibration control mount for UAV. The system consisted of a composite plate embedded with the magnets. The piezoelectric stack actuators were used to displace the plate and compensate for the disturbances. Proportional-integral-derivative controller was used to generate the control input to the piezoelectric

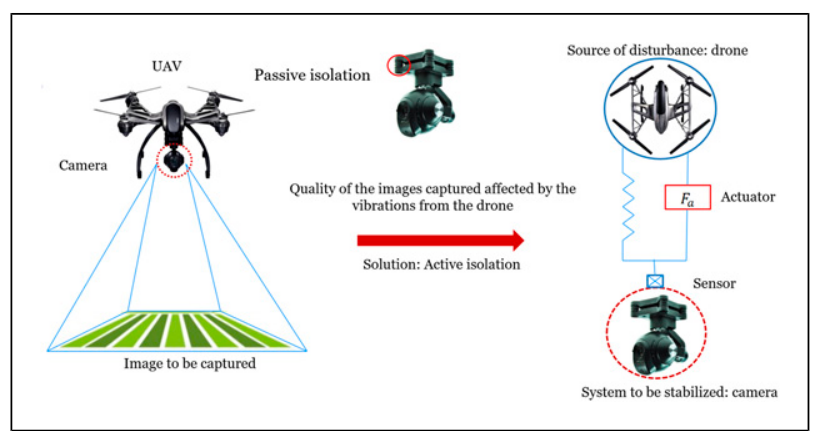

Figure 2. Concept of active stabilization of unmanned aerial vehicle imaging platform. The image quality is affected by the drone vibrations. Passive mounts are able to damp the resonance but degrades the response to high frequency. The proposed active isolation is able to damp the resonance without degrading the highfrequency response. 
actuator. Marichal et al. (2014) presented an active stabilization system using servo motors with neuro-fuzzy controller.

This article presents the studies carried out towards the development of an active isolation system for UAV imaging platform using contact-less voice-coil actuators. The active isolation system consists of an actuator at the center, supported by four vertical springs. The compact structure and the modular nature of the proposed isolation system make it pertinent to a wide range of drones and not just specific to a particular drone selected for the present study. The performance of the isolation system is investigated on a real drone camera subjected to the recorded drone spectrum. The organization of the article is as follows. The characterization of the drone vibrations are presented in Section 2. The tests carried out to study the effect of vibration on the quality of the images captured by the camera are presented in Section 3. The motivation and evolution of the proposed configuration of the isolation system are presented in Section 4. The controller design and active vibration control experiments are presented in Section 5, followed by concluding remarks in Section 6.

\section{Characterization of drone vibrations}

The first step is to characterize the nature of vibrations that are being transferred from the drone to the camera. The
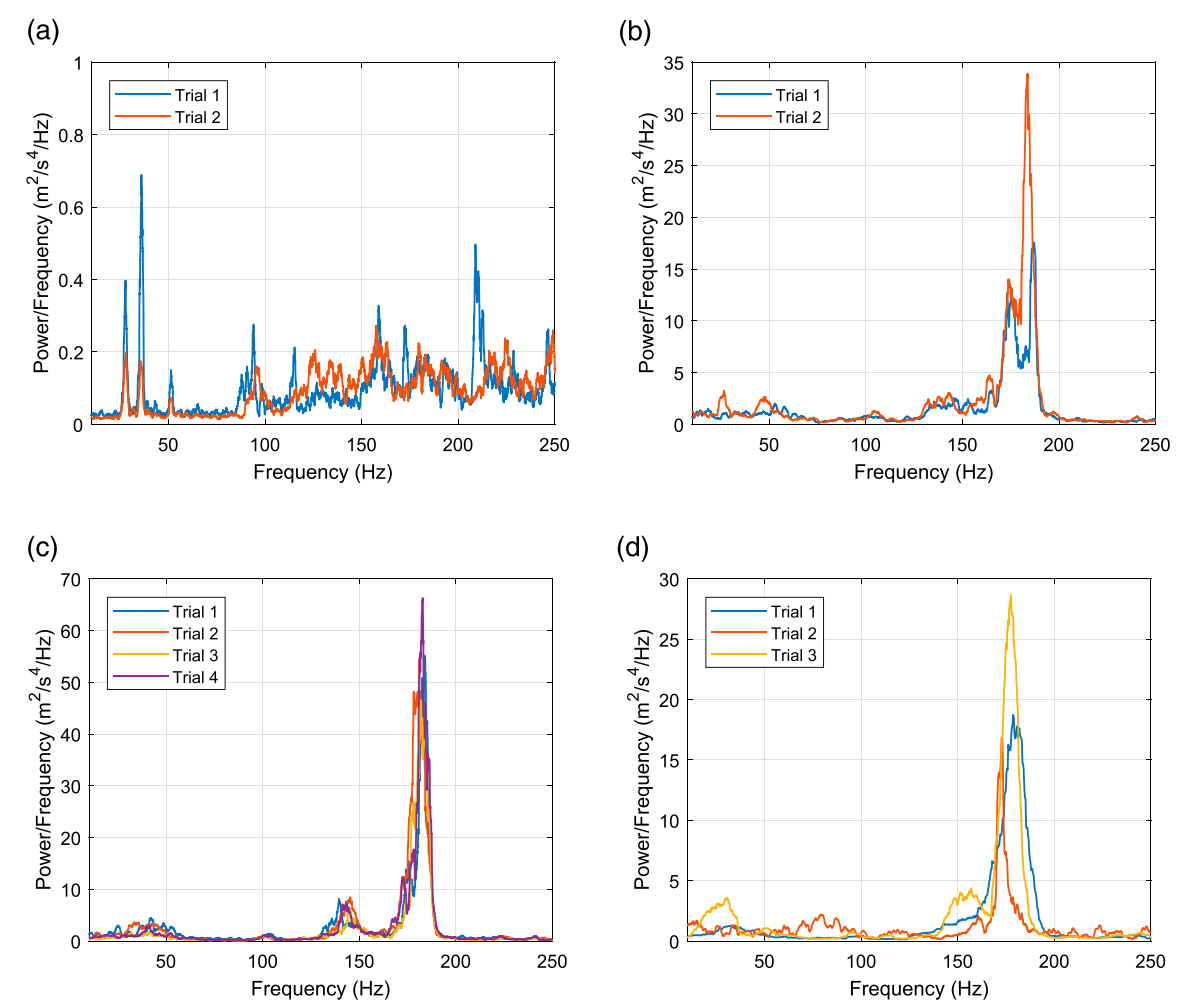

(e)

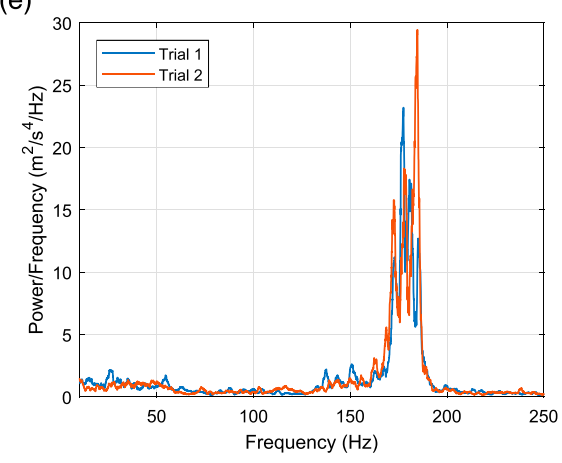

Figure 3. Power spectral density of the recorded acceleration data in different flight modes of the drone: (a) ground, (b) ascent, (c) hover, (d) descent and (e) in-flight. The drone vibrations are most significant in the hover mode around I50-200 Hz. 
experiments were conducted on a quad copter, and the acceleration time history was recorded. A wireless acceleration sensor was used to allow for the unrestricted movement of the drone. Use of the wireless sensor also eliminates the effect of the parasitic stiffness that may be added because of the wiring of the instrumentation. The acceleration was recorded only for vertical direction as the acceleration levels are most significant in this direction. The sampling frequency of the sensor was $512 \mathrm{~Hz}$. The acceleration data recorded were transferred to a local storage device using a wireless connection.

Acceleration data were recorded in five different modes to identify the critical acceleration levels.

1. Ground mode: drone is switched on but does not take off

2. Ascent mode: drone during take off

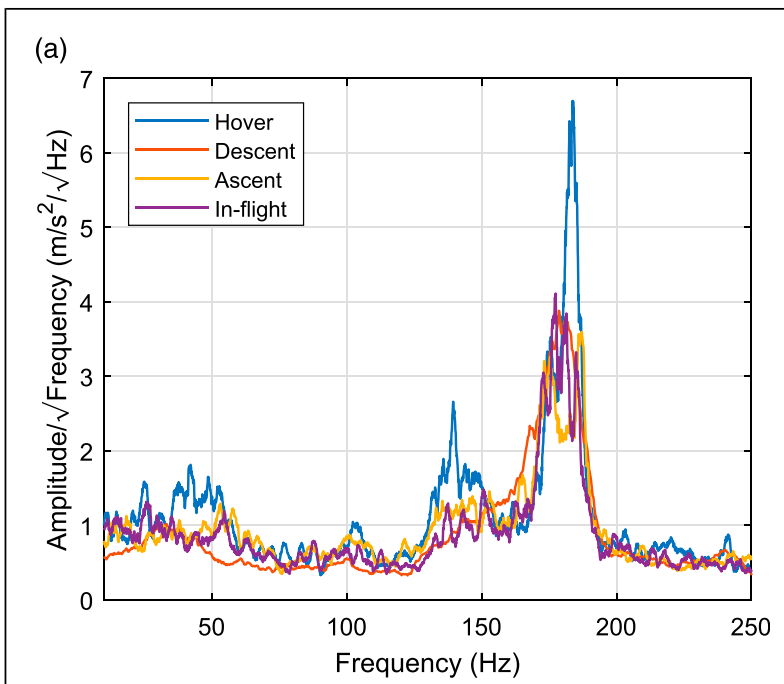

(b)

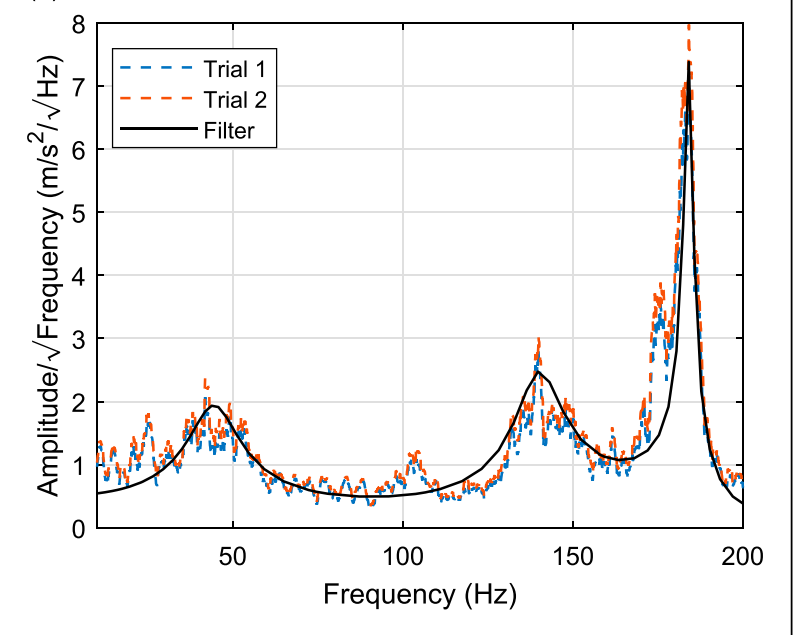

Figure 4. Comparison of amplitude spectral density of the drone vibration (a) in different flight modes and (b) in the hover mode with that of the designed filter. The designed filter matches well with the spectrum of drone vibrations in the hover mode and can be used to simulate the spectrum in laboratory environment.
3. Hover mode: drone stays in the steady position

4. Descent mode: drone during landing

5. In-flight mode: drone during flight

The power spectral densities (PSDs) of the acceleration data recorded for different modes are shown in Figure 3. It can be observed that there is a prominent peak around 175$185 \mathrm{~Hz}$. This corresponds to the rotational frequency of the motors. When the drone is on ground mode, there are two distinctive peaks at around 27 and $35 \mathrm{~Hz}$. This should correspond to the internal resonances of the drone. Acceleration levels are found to be maximum in the hover mode. The spectrum corresponding to the drone vibration was then simulated in the laboratory using a mechanical shaker. The comparison of the amplitude spectral density (ASD) of the drone acceleration in hover, descent, ascent, and in-flight modes is shown in Figure 4(a). It is observed that the drone acceleration is highest in the hover mode. Therefore, the spectrum corresponding to the drone acceleration in the hover mode is selected for simulation in the laboratory. The transfer function of the filter used for simulating the drone spectrum is as follows

$$
\begin{aligned}
& G_{f}=\frac{4.28 \times 10^{10}(s+995.8)^{2}}{\left(s^{2}+96.13 s+8 \times 10^{4}\right)\left(s^{2}+87.97 s+7.74 \times 10^{5}\right)} \\
& \times\left(s^{2}+16.19 s+1.34 \times 10^{6}\right)
\end{aligned}
$$

The ASDs of the drone acceleration in the hover mode and the designed filter are compared in Figure 4(b). It can be observed that the filter is able to simulate a vibration profile similar to that of the drone satisfactorily.

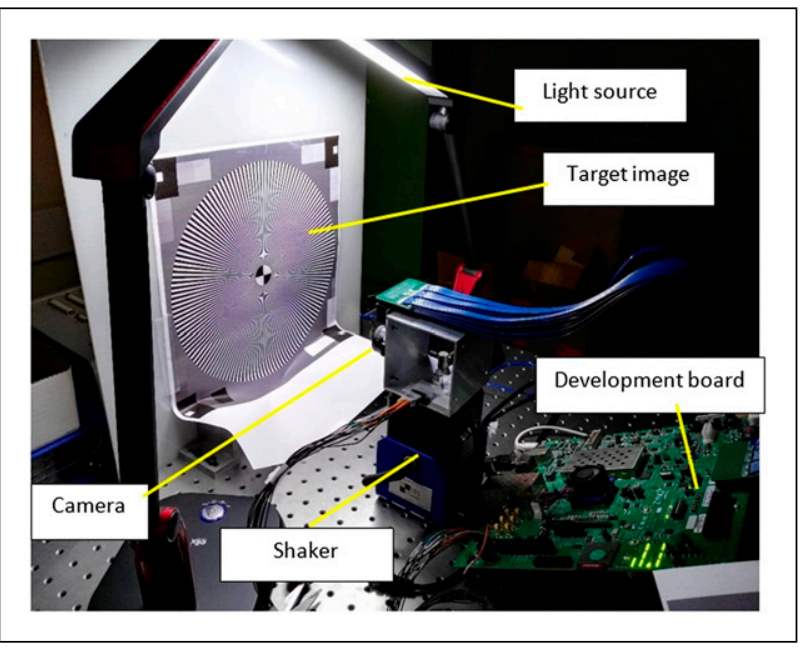

Figure 5. Test setup for studying the effect of vibrations on the quality of the recorded video signal. The camera is subjected to vibrations with the help of the shaker. The video stream is captured, and the image processing is carried out on a development board to calculate the PSNR. 


\section{Effect of vibrations on image quality}

Vibrations of the drone can severely affect the quality of the images/videos being captured by the drone. Experiments were conducted to study the effect of different vibration profiles on the quality of the video signals recorded. A test bench developed for this purpose is shown in Figure 5. The test setup consisted of two light-emitting diode light sources, a mechanical shaker, a target image, a camera system, and a development board (for image processing). A rigid box section was selected for mounting the camera. High stiffness of the mount ensures that the vibrations from the mechanical shaker are directly transferred to the camera. The camera was then rigidly attached to the mount. An accelerometer was installed on the mount to record the input acceleration. The designed filter was implemented using dSPACE MicroLabBox. Peak signal to noise ratio (PSNR) was used as a measure to quantify the effect of vibrations on the quality of the video signal. The camera was subjected to two different vibration profiles - white noise and drone spectrum. The results of the experiments are summarized in Table 1. Each test was conducted for three different trials. The drone spectrum corresponds to the acceleration spectrum of the drone in the hover mode simulated using the filter designed in equation (1). The gain was changed to achieve the different levels of vibrations. White noise corresponds to the random signal having uniform intensities at different frequencies. The variation of mean PSNR (mean of the PSNR obtained from three different trials-T1, T2, and T3) with the different gains for drone spectrum and white noise vibration profiles are shown in Figure 6. The PSNR is found to decrease with the increasing gain. The vibration levels are directly proportional to the gain used in the vibration model. This implies that the quality of the video signal is affected by the level of vibration that the camera experiences. Increased level of vibration results in poor quality of the video signal being captured.

Table I. Mean and variance of the PSNR recorded for different vibration profiles.

\begin{tabular}{|c|c|c|c|c|c|c|c|c|}
\hline \multirow[b]{2}{*}{ Test no. } & \multirow[b]{2}{*}{ Experiment name } & \multirow[b]{2}{*}{ Gain } & \multicolumn{3}{|c|}{ Mean PSNR } & \multicolumn{3}{|l|}{ Variance } \\
\hline & & & TI & $\mathrm{T} 2$ & T3 & TI & $\mathrm{T} 2$ & T3 \\
\hline I & Static & 0 & $4 I .4 I$ & 41.39 & $4 I .4 I$ & 0.0011 & 0.0053 & 0.0013 \\
\hline 2 & Drone spectrum I & 0.5 & 30.27 & 30.67 & 30.53 & 19.83 & 20.07 & 18.32 \\
\hline 3 & Drone spectrum 2 & 0.7 & 28.09 & 27.91 & 27.47 & 20.10 & 16.81 & 15.49 \\
\hline 4 & Drone spectrum 3 & 1.0 & 25.65 & 25.70 & 25.52 & 17.98 & 17.48 & 17.99 \\
\hline 5 & Drone spectrum 4 & 2.0 & 21.25 & 20.07 & 20.34 & 16.63 & $|3.3|$ & 13.44 \\
\hline 6 & Drone spectrum 5 & 0.3 & 32.79 & 32.87 & 34.21 & 15.13 & $12.7 \mid$ & 15.69 \\
\hline 7 & Drone spectrum 6 & 0.1 & 38.97 & 39.22 & 39.67 & 3.87 & 3.28 & 2.32 \\
\hline 8 & Drone spectrum 7 & 0.05 & 40.58 & 40.46 & 40.98 & 0.97 & 0.87 & 0.36 \\
\hline 9 & White noise I & 0.01 & 37.99 & 38.15 & 37.33 & 8.30 & 7.07 & 8.05 \\
\hline 10 & White noise 2 & 0.05 & 26.47 & 27.33 & 26.82 & 19.40 & 20.47 & 19.35 \\
\hline II & White noise 3 & 0.1 & 20.08 & 21.85 & 22.01 & 16.87 & 18.24 & 14.08 \\
\hline 12 & White noise 4 & 0.005 & 39.77 & 40.32 & 40.14 & 2.16 & 1.45 & 1.60 \\
\hline
\end{tabular}

PSNR: peak signal-to-noise ratio.

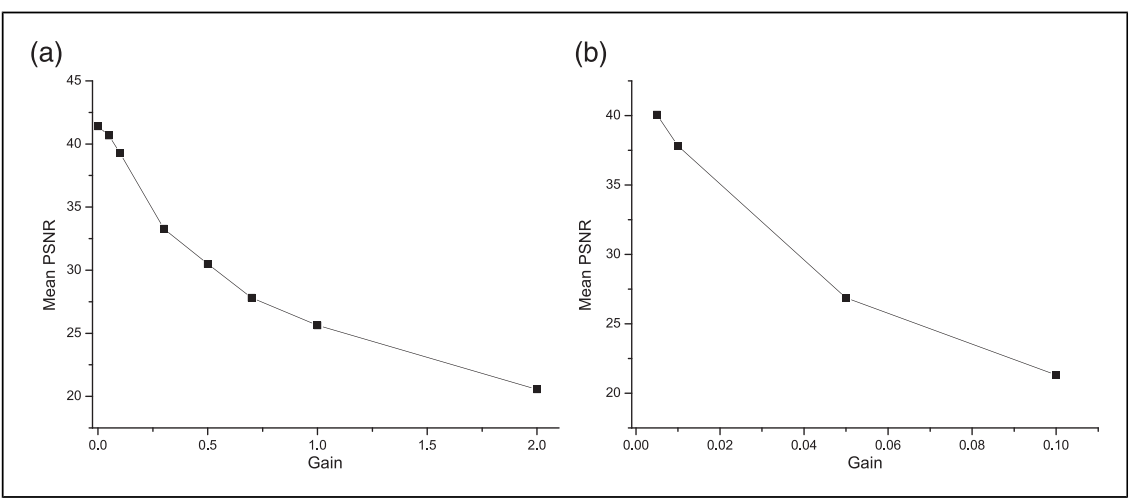

Figure 6. Variation of the mean peak signal-to-noise ratio (mean of TI, T2, and T3) with the gain for (a) drone spectrum and (b) white noise vibration profiles. It is observed that the quality of video/images captured is degraded with the increase in the vibrations. 


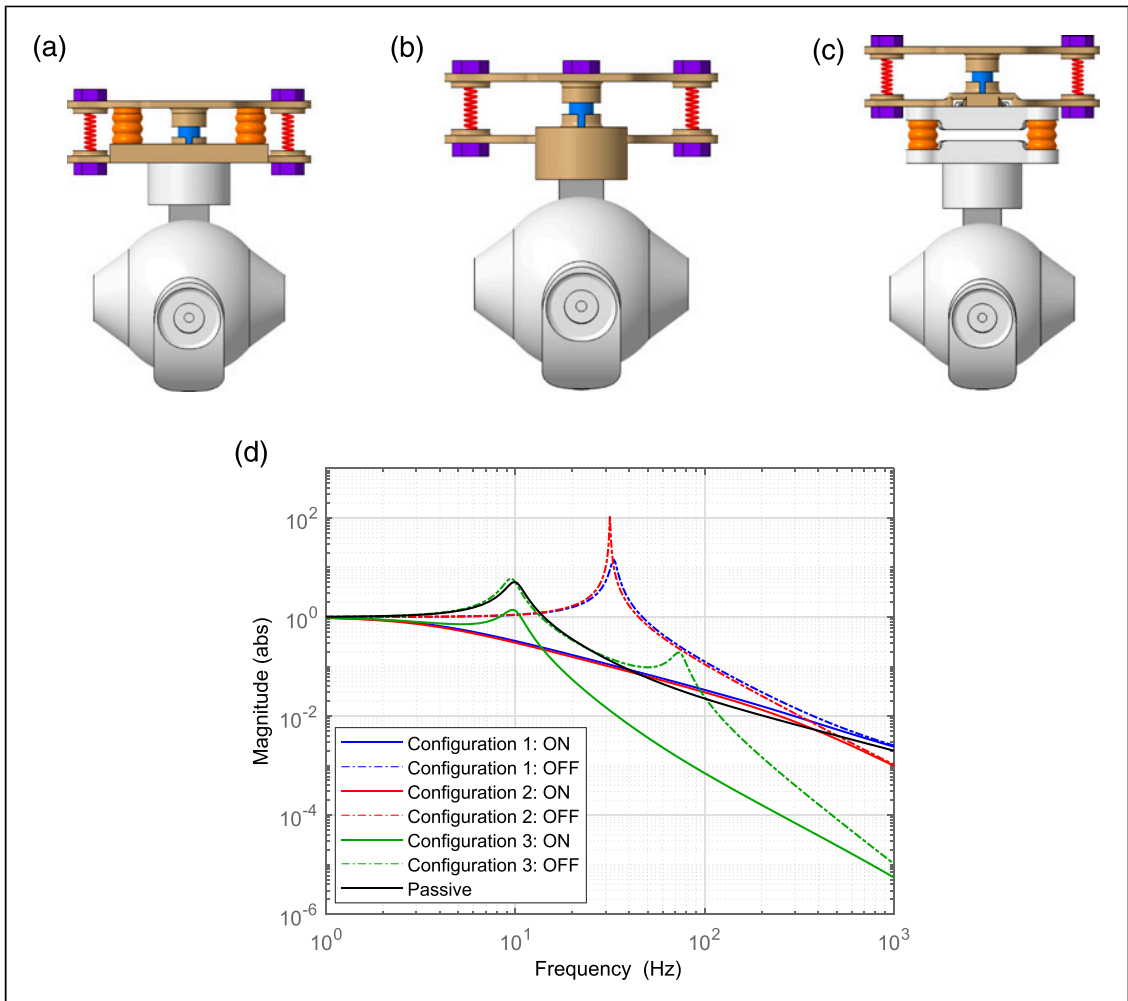

Figure 7. Various configurations of the isolation system based on the placement of active elements: (a) active element is integrated with the passive isolation, (b) passive isolation is removed and only active isolation is used, (c) isolation is performed in two stages, active element followed by passive isolation, and (d) comparison of the frequency response. The performance is better than the conventional system even when the control is off.

\section{Development of isolation system}

This section describes the steps followed to arrive at the configuration of the isolation system. This section also presents a numerical model of the isolation system developed based on the experiments. The same model will be used in the next section for designing the controller.

\section{I. Layout}

Preliminary studies are carried out to arrive at the configuration of the isolation system. The configuration can be varied depending on the placement of the actuators in the isolation system. The aim of this exercise is to have a qualitative idea about the performance of different configurations. The values used for the simulation are not exact and are arrived based on the preliminary design. To study the different configurations, the camera is idealized as rigid mass (equal to $0.5 \mathrm{~kg}$, obtained by weighting), whereas the passive rubber isolation is represented by a spring-dashpot system. The natural frequency of the camera with the passive isolation system is taken as $10 \mathrm{~Hz}$, and the damping ratio is assumed to be 0.1 (Bartel et al., 2018; Verma and Collette, 2019). Based on the preliminary design, The stiffness and damping associated with the active element (actuator) are assumed to be 10 and 0.1 times that of the passive isolation, respectively.

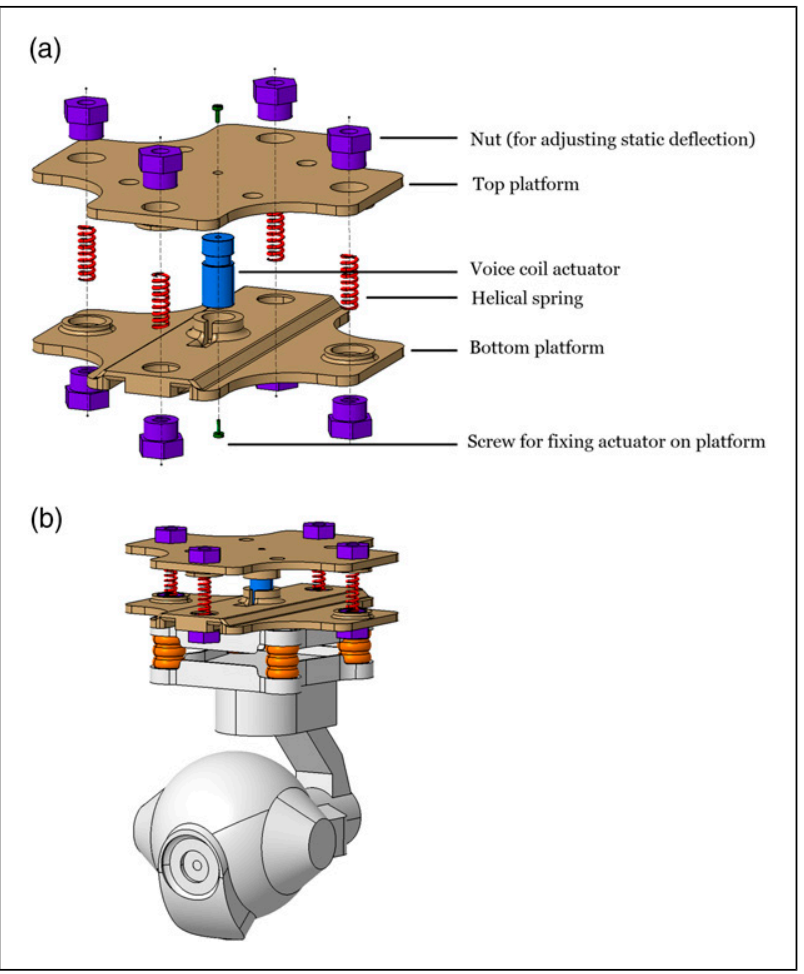

Figure 8. Schematics of the proposed isolation system: (a) exploded view of the active isolation system and (b) full assembly. 


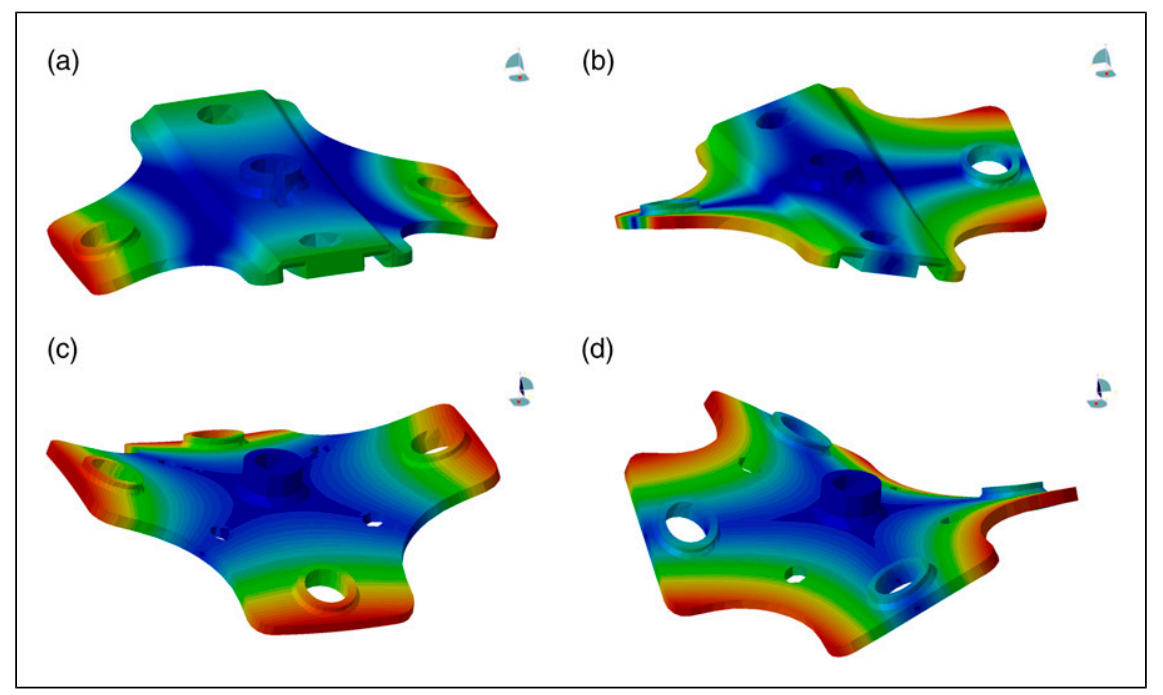

Figure 9. Flexible modes of top and bottom platform: (a) top platform; first mode, (b) top platform; second mode, (c) bottom platform; first mode, and (d) bottom platform; second mode. The flexible modes of the isolation platform are far from the frequency range of drone excitation, $150-200 \mathrm{~Hz}$.

Three different isolation configurations are considered as shown in Figure 7. In first configuration (Figure 7(a)), the active isolation mounts are integrated with the conventional passive rubber mounts. In the second configuration (Figure 7(b)), the passive rubber mounts are removed and only active elements are used in the isolation system. In third configuration (Figure 7(c)), the isolation is carried out in two stages. The active elements are first integrated on an intermediate isolation platform (whose mass is assumed to be one-fifth of the mass of the camera for preliminary analysis) followed by passive isolation rubber mounts. Acceleration feedback is used for active control. For each of these configurations, the controller used is simply an integrator with a gain of 1000 (i.e. 1000/s). Frequency responses for various configurations are compared in Figure 7(d). It can be observed that configurations 1 and 2 are effective in reducing the response of the camera below $50 \mathrm{~Hz}$ but are not effective in reducing the response of the camera about $100 \mathrm{~Hz}$ compared with the passive isolation. Because the region of interest in our application is above $100 \mathrm{~Hz}$ (refer Section 2, the magnitude of the drone vibrations is more significant for the frequencies above $100 \mathrm{~Hz}$ ), the configurations 1 and 2 are not useful. Moreover, they are found to magnify the response when the control is off. The third configuration is found to perform much better than the other two configurations. It is found to significantly reduce the high-frequency response. Even when the control is off, it performs better than conventional passive isolation and, hence, can be considered as fail-safe. The third configuration is taken up for further consideration in the rest of the article.

\subsection{Construction material}

The design of the isolation system is also limited by the weight of the platform. The platform cannot be too heavy as

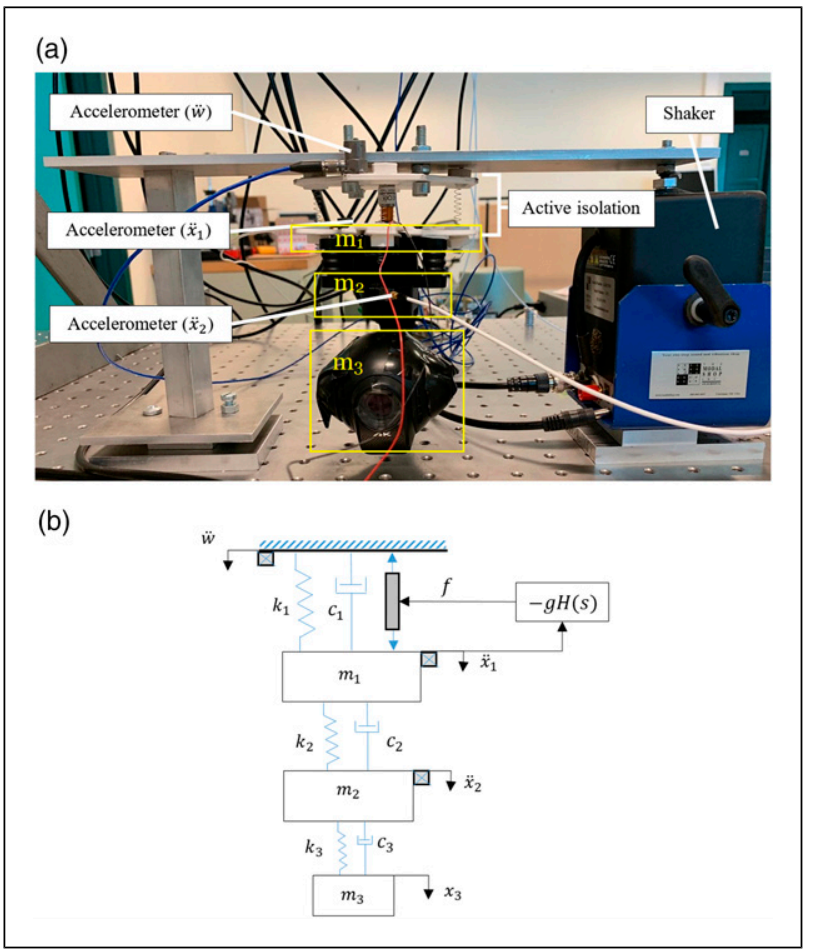

Figure 10. Experimental investigations: (a) test setup; the shaker is used for injecting external disturbances due to the drone and the acceleration response is recorded at three different locations and (b) idealized model of isolation-camera system. Acceleration response of the bottom platform is used as a feedback.

it will result in a higher payload. The drone may not be able to fly with a high payload. Also, one needs to comply with the guidelines on the allowable weight laid down by the air traffic control authority. One of the ways to achieve the 
light weight design is by using additive manufacturing techniques. Three-dimensional printing of the actuator housing assembly using polylactic acid (PLA) offers various advantages over other materials. Parts made from PLA are light weight and have high toughness, resilience, and durability.

\subsection{Components}

The guides in voice coil actuators are neither perfectly rigid nor massless. The dynamics of the guides can interfere with those of the isolator and can significantly affect the transmissibility of the isolator at frequencies beyond the resonance (Preumont et al., 2007). This problem is overcome in the current design by using a contact-less voice coil actuator. Because there is no stiffness associated with the active element, the issue of spurious resonance is nonexistent anymore. The contact-less voice coil actuators are guided by low-stiffness stainless steel compression springs. The static deflection of the isolation system when the camera is attached needs to be adjusted to ensure that the voice coil actuator stays in the mid-stroke position. For this purpose, a special type of fastener is designed, which allows adjustment of the static deflection. The final assembly of the isolation system is shown in Figure 8.

\subsection{Flexible modes}

The isolation system should be designed carefully to avoid local resonances due to high-frequency vibrations (above $150 \mathrm{~Hz}$ ) coming from the drone. After designing the assembly, a finite element analysis is carried out to identify the flexible modes and to ensure that these modes are far from frequency of excitation. The first two flexible modes of the top and bottom platform of the isolation system are shown in Figure 9.

\section{Experimental investigations}

This section presents the details of the setup, system identification, controller design, and active control experiments carried out with the proposed isolation system.

\section{I. Setup}

The test setup used for experimental investigations is shown in Figure 10(a). The setup consists of a cantilever beam
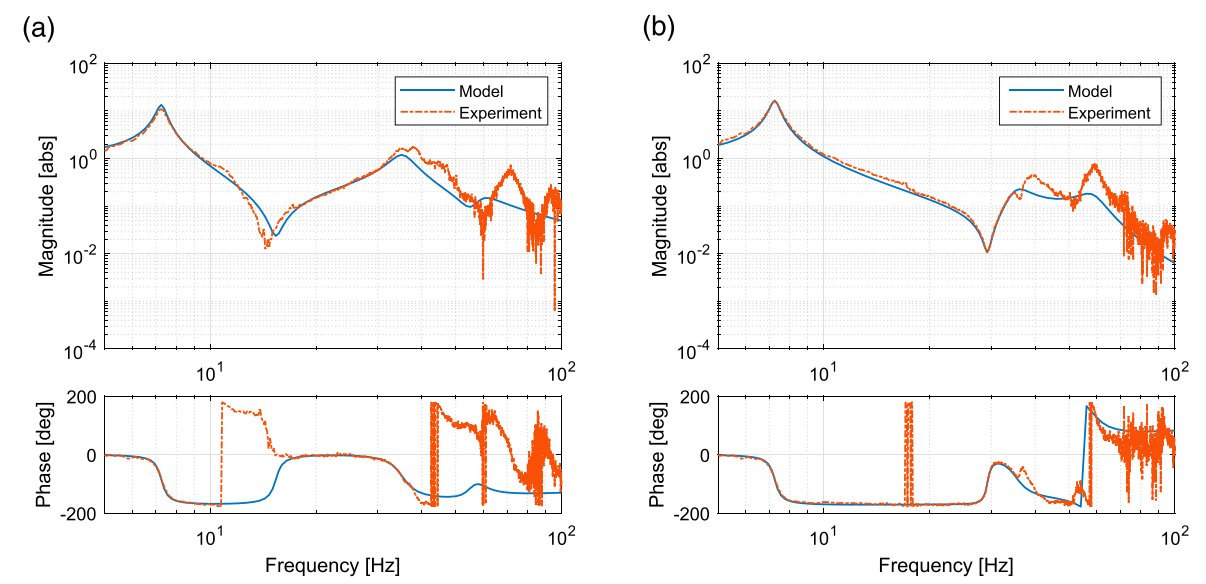

(c)
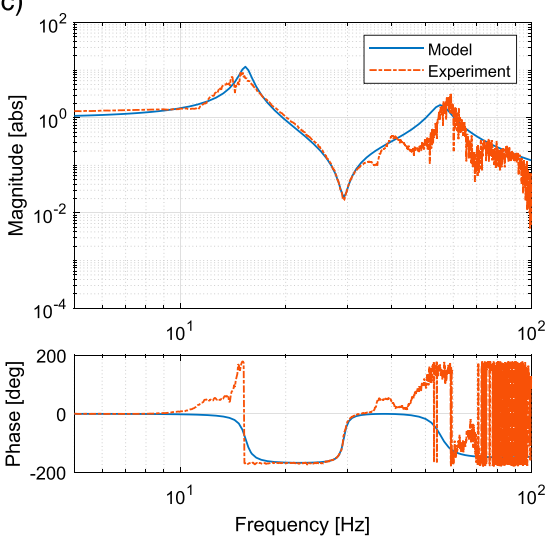

Figure I I. Comparison of the frequency response obtained from the experiments with the model: (a) $\ddot{w}$ to $\ddot{\mathbf{x}}_{1}$, (b) $\ddot{\mathbf{w}}$ to $\ddot{\mathbf{x}}_{2}$, and (c) $\ddot{\mathbf{x}}_{1}$ to $\ddot{\mathbf{x}}_{2}$. In all the figures, the response of the model is found to match well with the experiments. 
whose free end is supported on a shaker. The isolation system and the camera unit are mounted under this beam. The external disturbances are injected via the shaker. The response of the isolation system at different levels is recorded using accelerometers. The controller is implemented in real time using dSpace MicroLabBox. The current injector is used to drive the voice coil actuator with the input signal received from the controller.

\subsection{System identification}

The first step of the control design is to identify the properties of the system to be controlled. The frequency response of the system is evaluated experimentally by injecting white noise into the system using the shaker. From the frequency response obtained, it was found that the system essentially behaves like a three-degree-of-freedom system. The idealized system is shown in Figure 10(b).

The dynamic equation of the system can be written as

$$
\mathbf{M} \ddot{\mathbf{x}}+\mathbf{C} \dot{\mathbf{x}}+\mathbf{K x}=-\mathbf{M} \ddot{\mathbf{w}}+\mathbf{E f}
$$

where $\mathbf{M}$ is the mass matrix, $\mathbf{C}, \mathbf{K}$ are the stiffness and damping matrices, $\mathbf{E}$ is the matrix describing the location of the actuator, $\mathbf{x}$ is the motion vector of the platform relative to the base, $\mathbf{w}$ is the motion imposed at the base of the isolation, and $\mathbf{f}$ is the control force. The mass, damping, stiffness, and location matrices of the isolation platform are equal to

$$
\begin{aligned}
& \mathbf{M}=\left[\begin{array}{ccc}
m_{1} & 0 & 0 \\
0 & m_{2} & 0 \\
0 & 0 & m_{3}
\end{array}\right], \mathbf{K}=\left[\begin{array}{ccc}
k_{1}+k_{2} & -k_{2} & 0 \\
-k_{2} & k_{2}+k_{3} & -k_{3} \\
0 & -k_{3} & k_{3}
\end{array}\right], \\
& \mathbf{C}=\left[\begin{array}{ccc}
c_{1}+c_{2} & -c_{2} & 0 \\
-c_{2} & c_{2}+c_{3} & -c_{3} \\
0 & -c_{3} & c_{3}
\end{array}\right], \quad \mathbf{E}=\left[\begin{array}{l}
1 \\
0 \\
0
\end{array}\right]
\end{aligned}
$$

The values of the parameters in the mass, stiffness, and damping matrices are tuned to match with the experimental response. The comparison of the frequency response obtained from the experiments with that obtained from the model is shown in Figure 11. It can be observed that a good match is obtained between the experiments and the model.

\subsection{Controller design}

Inertial control is used in the present study to actively damp the resonances of the isolation system. The state-space form of the isolation system which takes $\ddot{\mathbf{w}}, \mathbf{f}$ as the inputs and gives inertial acceleration, $\overline{\mathbf{z}}=\left[\ddot{\mathbf{x}}_{1}, \ddot{\mathbf{x}}_{2}, \ddot{\mathbf{x}}_{\mathbf{3}}\right]^{\mathbf{T}}$, as the output can be written as

$$
\begin{aligned}
& \dot{\mathbf{z}}=\mathbf{A z}+\mathbf{B}_{\mathbf{f}} \mathbf{f}+\mathbf{B}_{\mathbf{w}} \ddot{\mathbf{w}} \\
& \overline{\mathbf{z}}=\mathbf{C}_{\mathbf{z}} \mathbf{z}+\mathbf{D}_{\mathbf{f}} \mathbf{f}+\mathbf{D}_{\mathbf{w}} \ddot{\mathbf{w}}
\end{aligned}
$$

where

$$
\begin{aligned}
& \mathbf{A}=\left[\begin{array}{cc}
\mathbf{0} & \mathbf{I} \\
-\mathbf{M}^{-1} \mathbf{K} & -\mathbf{M}^{-1} \mathbf{C}
\end{array}\right], \quad \mathbf{B}_{\mathbf{f}}=\left[\begin{array}{c}
\mathbf{0} \\
\mathbf{M}^{-1} \mathbf{E}
\end{array}\right], \\
& \mathbf{B}_{\mathbf{w}}=\left[\begin{array}{c}
\mathbf{0} \\
-\mathbf{I}
\end{array}\right], \quad \mathbf{z}=\left[\begin{array}{l}
\mathbf{x} \\
\dot{\mathbf{x}}
\end{array}\right], \\
& \mathbf{C}_{\mathbf{z}}=\left[\begin{array}{lll}
-\mathbf{M}^{-1} \mathbf{K} & -\mathbf{M}^{-1} \mathbf{C}
\end{array}\right], \quad \mathbf{D}_{\mathbf{f}}=\mathbf{0}, \quad \mathbf{D}_{\mathbf{w}}=\mathbf{0}
\end{aligned}
$$

The inertial acceleration of the bottom plate $\left(\ddot{\mathbf{x}}_{\mathbf{1}}\right)$ of the isolation platform is used as a feedback. This signal is integrated to generate a signal similar to the damping force.

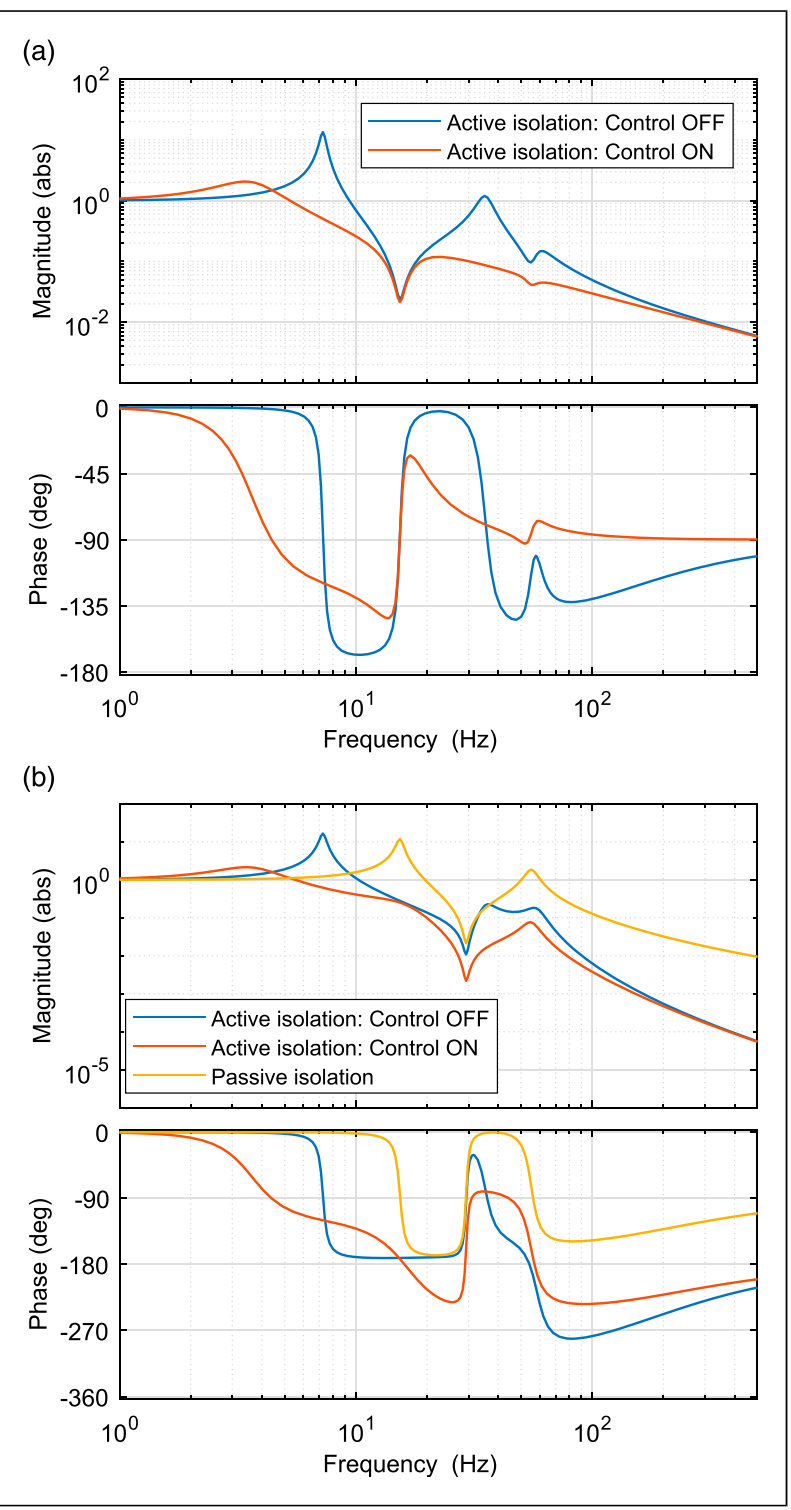

Figure 12. Comparison of the closed loop performance of the active isolation system from: (a) $\ddot{\mathbf{w}}$ to $\ddot{\mathbf{x}}_{1}$ and (b) $\ddot{\mathbf{w}}$ to $\ddot{\mathbf{x}}_{2}$. Notice that the response of the proposed isolation is almost 100 times less than the passive system around $150-200 \mathrm{~Hz}$. 
To avoid drift and actuator saturation, pure integrator is replaced by a weak integrator with a cutoff frequency at $5 \mathrm{~Hz}$. The transfer function of the controller used is

$$
H(s)=\frac{2 \pi \cdot 5}{s+2 \pi \cdot 5}
$$

The control force is given by

$$
\mathbf{f}=-g H(s) \cdot \ddot{\mathbf{x}}_{\mathbf{1}}
$$

where $g$ is the gain of the controller. The designed controller can either be implemented as an analog circuit using an operational amplifier or digitally using a microcontroller (e.g. Arduino pro, Arduino pro mini or micro, and Teensy 3.1). It should be noted that the sensor-actuator pair in the proposed isolation system are collocated. The system has alternating poles and zeros which provide infinite gain margin. The performance of the closed loop system for $g=1$ is shown in Figure 12. It can be observed that using inertial control we are able to damp the resonances without compromising on the performance at high frequency. The response between 150 and $200 \mathrm{~Hz}$ (which is the bandwidth where the drone excitation is dominant) is about 100 times less with the active isolation system than that with the passive isolators (see Figure 3).

\subsection{Frequency domain response}

After designing the controller, experiments are conducted to see the effect of the control on the isolation performance. A white noise is injected using the shaker. The response of the isolation system is recorded without control and with inertial control. The frequency response obtained from the experiments is compared in Figure 13. It can be observed that the inertial control is effective in damping the resonances of the isolation system. The PSD and cumulative root mean square (RMS) (cumulative over high to low frequency) of the acceleration, $\ddot{\mathbf{x}}_{\mathbf{2}}$, when subjected to recorded drone spectrum are compared in Figure 14(a) and

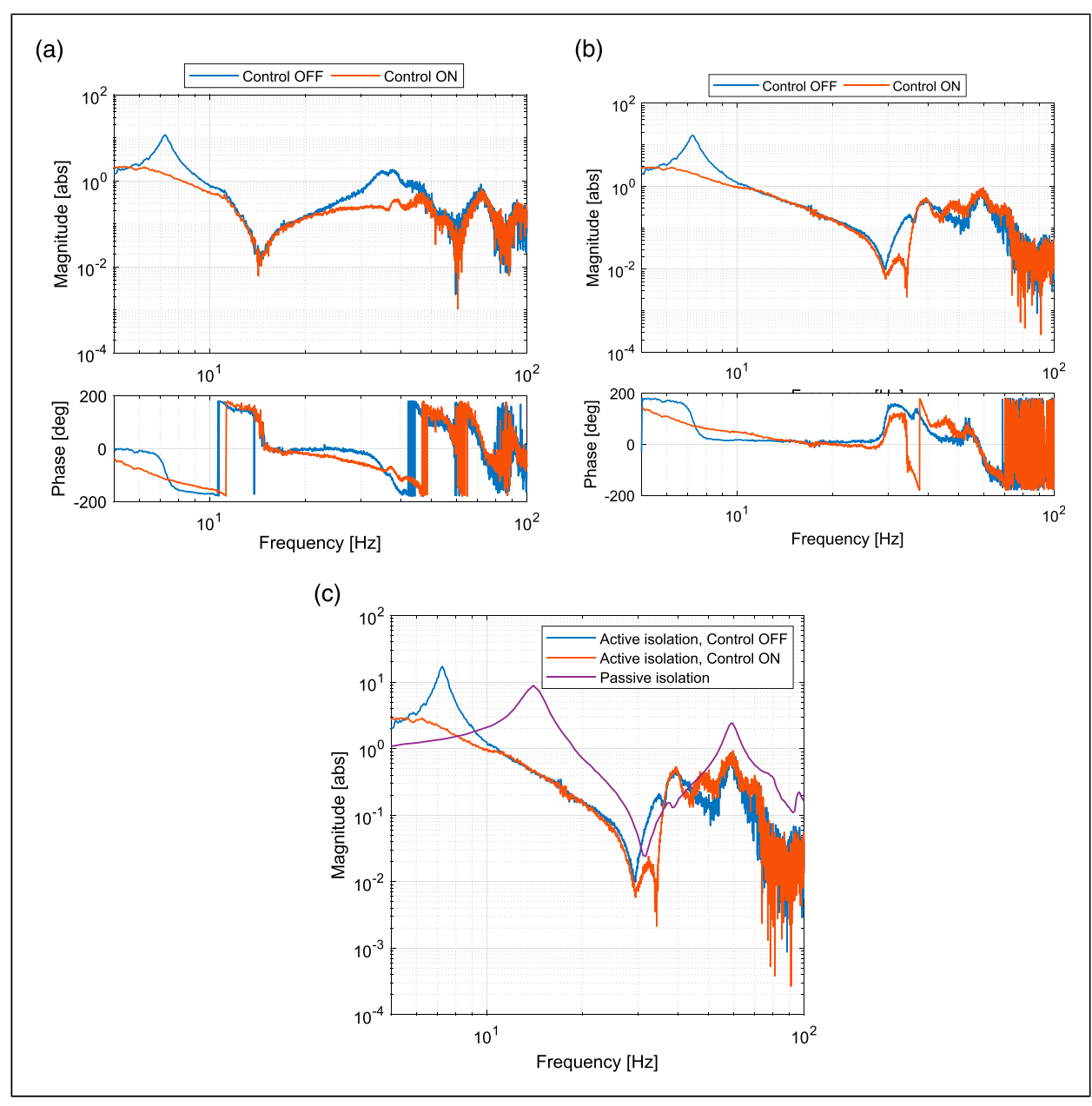

Figure 13. Comparison of the experimental closed loop performance of the active isolation system from: (a) $\ddot{\mathbf{w}}$ to $\ddot{\mathbf{x}}_{\mathbf{l}}$ and (b) $\ddot{\mathbf{w}}$ to $\ddot{\mathbf{x}}_{\mathbf{2}}$. The inertial control is effective in damping the resonances without degrading the high-frequency response of the system and (c) comparison of the experimental frequency response of the active isolation with the passive (from $\ddot{\mathbf{w}}$ to $\ddot{\mathbf{x}}_{2}$ ). 


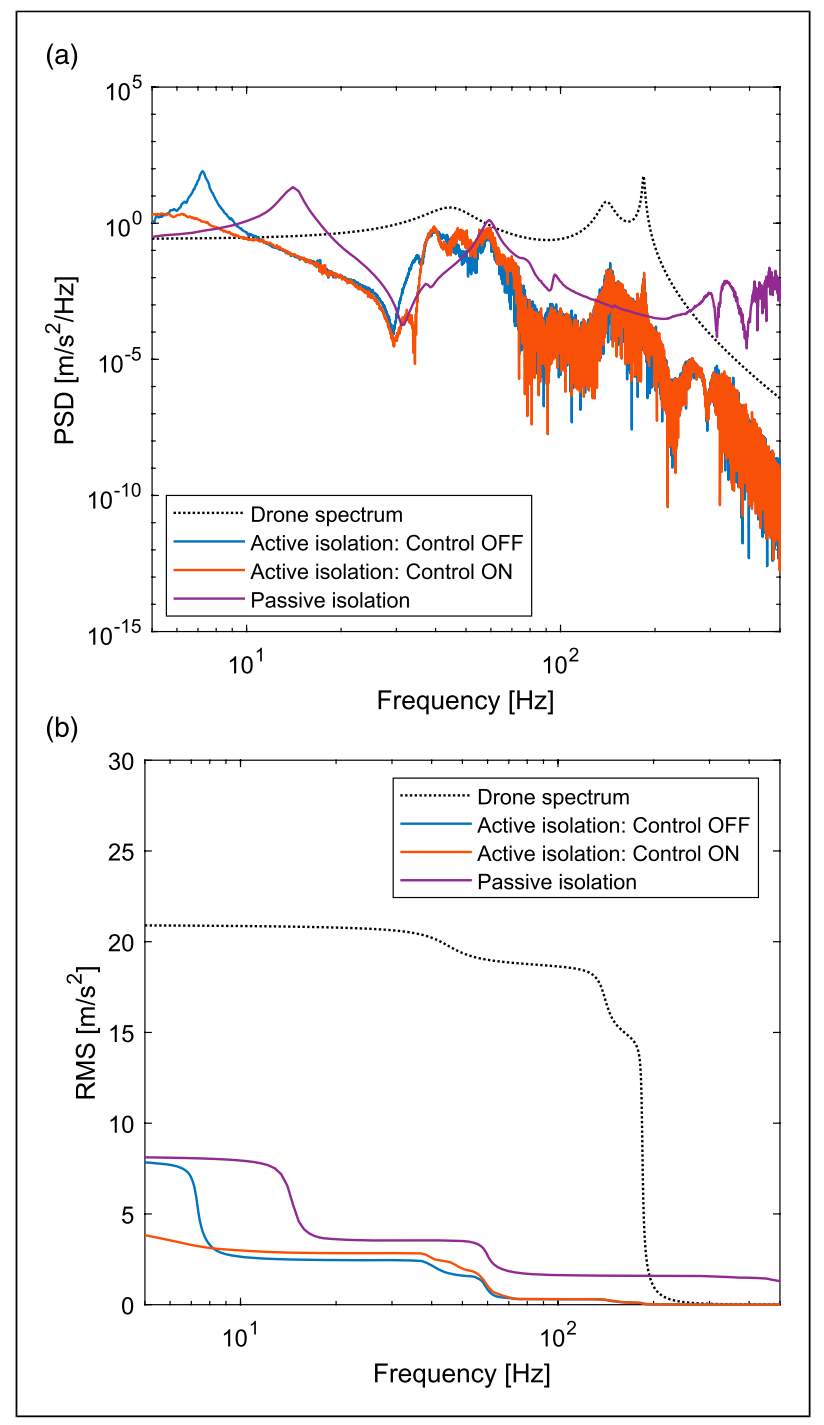

Figure 14. Comparison of the acceleration response $\left(\ddot{\mathbf{x}}_{\mathbf{2}}\right)$ for active and passive isolation when subjected to recorded drone spectrum: (a) power spectral density and (b) cumulative root mean square. Notice that the cumulative root mean square values are least for the proposed isolation system.

(b), respectively. The cumulative RMS, $\sigma_{\text {rms }}$, of the acceleration is calculated using the following equation

$$
\sigma_{\mathrm{rms}}(\omega)=\sqrt{\int_{\omega}^{\infty} T_{\ddot{\mathbf{x}}_{2} \ddot{\mathbf{w}}} \phi_{\ddot{\mathbf{w}}}(v) \cdot d v}
$$

where $T_{\ddot{\mathbf{x}}_{2} \ddot{\mathbf{w}}}$ is the transfer function from $\ddot{\mathbf{W}}$ to $\ddot{\mathbf{x}}_{\mathbf{2}}$ and $\phi_{\ddot{\mathbf{w}}}$ is the PSD of drone acceleration.

\subsection{Time-domain response}

The time-domain response of the active isolation system when subjected to the drone spectrum is evaluated experimentally. The filter designed in equation (1) is used to

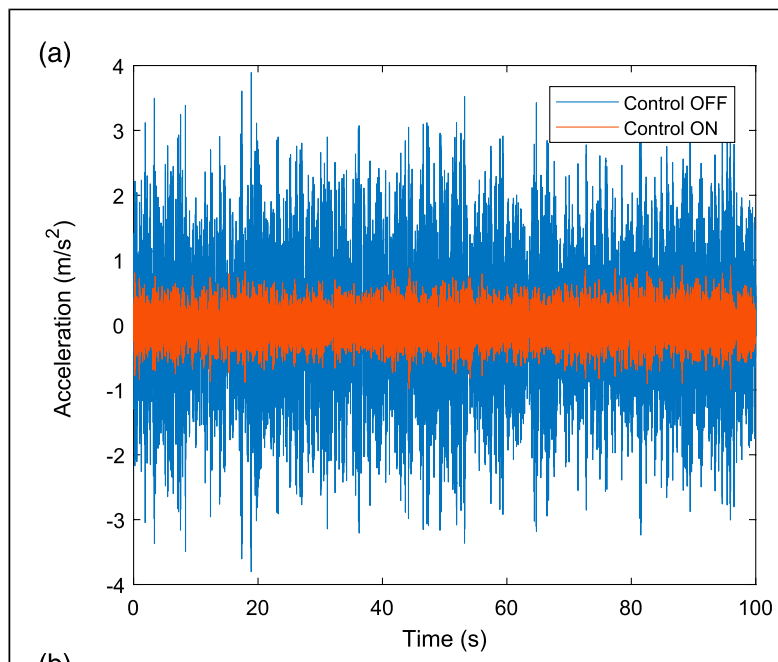

(b)

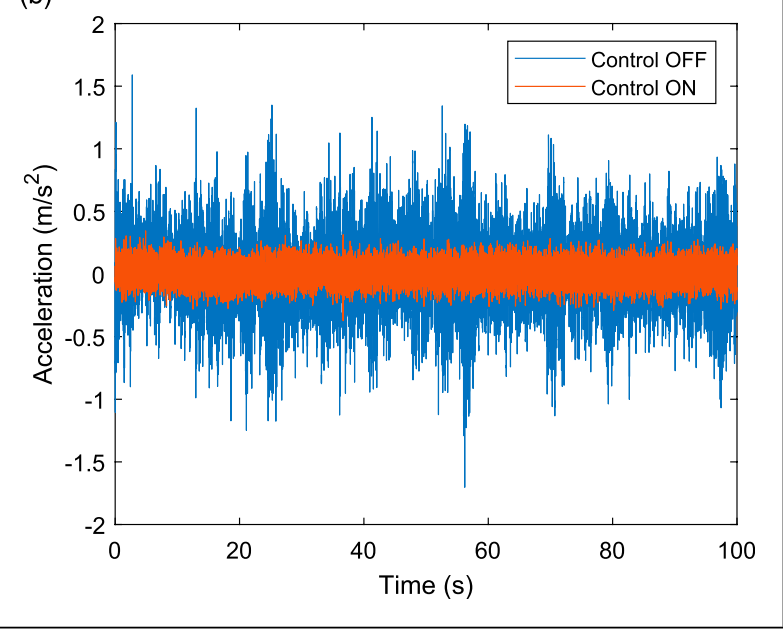

Figure 15. Comparison of the acceleration time history $\left(\ddot{\mathbf{x}}_{1}\right)$ of the active isolation with and without inertial control: (a) drone spectrum and (b) white noise. The acceleration levels are found to reduce to a factor of one-fourth (25\%) with the inertial control.

simulate a spectrum similar to the drone excitation in the laboratory. The excitation $\ddot{\mathbf{w}}$ is generated by passing white noise through the designed filter. The acceleration response at the bottom platform is recorded. The acceleration response is also recorded when the excitation is pure white noise. The comparison of the acceleration time history without control and with inertial control (with $g=5$ ) for two different excitation is shown in Figure 15. It can be observed that the inertial control is quite effective in reducing the acceleration levels at the bottom platform. The acceleration levels are reduced by almost $75 \%$.

\section{Concluding remarks}

The use of UAVs provides economic means to obtain images of which could be used for analysis, monitoring, and decision-making. However, the quality of images acquired 
is affected by the vibrations of the UAV which are high frequency and low amplitude in nature. Conventionally, passive rubber mounts are used to damp these vibrations. The passive system is good for damping the resonance but degrades the high-frequency response of the system. To address this issue, an active isolation system has been proposed in this article. The isolation system consists of a single contact-less voice coil actuator which is supported by four springs. The isolation is carried out in two stages. The active isolation is integrated between the drone and the conventional camera-passive isolation system. The top and bottom platform of the active isolation are 3D printed using PLA material. Special screws are designed to allow for the adjustment of the static deflection due to the weight of the camera. The isolation platform is designed in such a way that its flexible modes are away from the drone excitation frequency. Acceleration feedback is used to damp the resonance of the isolation system. Even when the controls are off, the isolation system is found to exhibit better performance than the conventional rubber mounts and, hence, can be considered as fail-safe. The performance of the system is validated experimentally in both frequency and time-domain. The active isolation system is found to exhibit better highfrequency response than in the conventional system. The active control is found to reduce the acceleration levels on the camera to one-fourth of the acceleration levels without control. The proposed isolation system is not specific to UAVs and, hence, can be used in various applications.

\section{Acknowledgements}

The authors gratefully acknowledge the Wallonie Region for funding this research (WALInnov Projet Trusteye, Grant No. 4.139.H.000170). The authors acknowledge the help provided by Alexandre Willeme (from Universite Catholique de Louvain, UCLouvain) and Laura Joris (from University of Liege, ULg) in conducting the experiments.

\section{Declaration of conflicting interests}

The author(s) declared no potential conflicts of interest with respect to the research, authorship, and/or publication of this article.

\section{Funding}

The author(s) disclosed receipt of the following financial support for the research, authorship, and/or publication of this article: The authors gratefully acknowledge the Wallonie Region for funding this research (WALInnov Projet Trusteye, Grant No. 4.139.H.000170).

\section{ORCID iD}

Mohit Verma (D) https://orcid.org/0000-0001-6026-0507

\section{References}

Baek J (2012) Course Material for CS 478, Computational Photography, Spring 2012. CA, USA: Stanford University.
Available at: http:/graphics.stanford.edu/courses/cs478/ lectures/03072012_image_stabilization.pdf.

Baer N and Semke WH (2012) A frictionless lightweight active vibration control mount for small uas. In: Topics in Modal Analysis I. NY, USA: Springer, Vol. 5, 367-375.

Bartel T, Atzrodt H and Wilczynski D (2018) Active vibration reduction on a quadrocopter. In: International conference on noise and vibration engineering, Leuven, Belgium, 17-19 September, 2018, pp. 131-141, Leuven, Belgium: ISMA 2018 KU Leuven.

Boike J and Yoshikawa K (2003) Mapping of periglacial geomorphology using kite/balloon aerial photography. Permafrost and Periglacial Processes 14(1): 81-85.

Chen YR, Chao K and Kim MS (2002) Machine vision technology for agricultural applications. Computers and Electronics in Agriculture 36(2-3): 173-191.

Gjika K and Dufour R (1999) Rigid body and nonlinear mount identification: application to onboard equipment with hysteretic suspension. Journal of Vibration and Control 5(1): 75-94.

Jin JS, Zhu Z and Xu G (2000) A stable vision system for moving vehicles. IEEE Transactions on Intelligent Transportation Systems 1(1): 32-39.

Kienholz DA, Smith CA and Haile WB (1996) Magnetically damped vibration isolation system for a space shuttle payload. In: Smart Structures and Materials 1996: Passive Damping and Isolation. CA, USA: International Society for Optics and Photonics, Vol. 2720, 272-281.

Levoy M (2014) Course material for CS 178, Optical image stabilization (IS), Spring 2014. CA, USA: Stanford University. Available at: https://graphics.stanford.edu/courses/cs178/ lectures/stabilization-29apr14.pdf.

Maes JL, Binczak S and Lhenry V (2014) A passive stabilization solution for camera embedded onboard small planes. In: 2014 Integrated communications, navigation and surveillance conference (ICNS), Herndon, VA, 8-10 April 2014, pp. U3-1. NJ, USA: IEEE.

Marichal GN, Tomás-Rodríguez M, Hernández Á, et al. (2014) Vibration reduction for vision systems on board unmanned aerial vehicles using a neuro-fuzzy controller. Journal of Vibration and Control 20(15): 2243-2253.

Ma'sum MA, Arrofi MK, Jati G, et al. (2013) Simulation of intelligent unmanned aerial vehicle (UAV) for military surveillance. In: 2013 International conference on advanced computer science and information systems (ICACSIS), Bali, Indonesia, 28-29 September 2013, pp. 161-166. NJ, USA: IEEE.

Oh JS, Choi SB, Cho HJ, et al. (2010) Performance evaluation on an active camera mount system for UAV via hardware-in-theloop-simulation. Transactions of the Korean Society for Noise and Vibration Engineering 20(8): 767-773.

Oh JS, Han YM and Choi SB (2011) Vibration control of a camera mount system for an unmanned aerial vehicle using piezostack actuators. Smart Materials and Structures 20(8): 085020.

Park DH and Choi SB (2008) Active vibration control of UAV EO/ IR sensor mount using piezoelectric actuator. Transactions of the Korean Society for Noise and Vibration Engineering 18(12): 1278-1285.

Pete AE, Kress D, Dimitrov MA, et al. (2015) The role of passive avian head stabilization in flapping flight. Journal of the Royal Society Interface 12(110): 20150508. 
Preumont A, Horodinca M, Romanescu I, et al. (2007) A six-axis single-stage active vibration isolator based on Stewart platform. Journal of Sound and Vibration 300(3-5): 644-661.

Stuckel KJ and Semke WH (2011) A piezoelectric actuated stabilization mount for payloads onboard small UAS. In: Advanced Aerospace Applications. NY, USA: Springer, Vol. 1, 295-305.

Stuckel KJ, Semke WH, Baer N, et al. (2011) A high frequency stabilization system for UAS imaging payloads. In: Structural Dynamics. NY, USA: Springer, Vol. 3, 1411-1419.

Verma $\mathrm{M}$ and Collette C (2019) Active vibration isolation system for drone cameras. In: International conference on vibration problems, ICVOP 2019, Crete, Greece, 1-4 September 2019, ULB: Belgium, Paper ID 18795.

Wahbeh AM, Caffrey JP and Masri SF (2003) A vision-based approach for the direct measurement of displacements in vibrating systems. Smart Materials and Structures 12(5): 785 .

Webster AL and Semke WH (2005) Broad-band viscoelastic rotational vibration control for remote sensing applications. Journal of Vibration and Control 11(11): 1339-1356.

Windau J and Itti L (2011) Multilayer real-time video image stabilization. In: 2011 IEEE/RSJ international conference on intelligent robots and systems (IROS), San Francisco, CA, 2530 September 2011, pp. 2397-2402. NJ, USA: IEEE.

Xiang H and Tian L (2011) Development of a low-cost agricultural remote sensing system based on an autonomous unmanned aerial vehicle (UAV). Biosystems Engineering 108(2): 174-190.

Yao Z and Jingrui Z (2015) The imaging stability enhancement of optical payload using multiple vibration isolation platforms. Journal of Vibration and Control 21(9): 1848-1865. 